\title{
Prenatal Diagnosis and Successful Palliation of Absent Aortic Valve with Hypoplastic Left Heart Syndrome: A Case Report and Review of Literature
}

\author{
Amna Qasim, MD ${ }^{1 *(\cdot)}$ Chelsea B. Johnson, MD ${ }^{1} \quad$ Muhammad A. Aly, BS ${ }^{1}$ Ashraf M. Aly, MD, PhD $2 *(\cdot)$ \\ ${ }^{1}$ Department of Pediatrics, University of Texas Medical Branch, \\ Galveston, Texas \\ 2 Division of Pediatric Cardiology, University of Texas Medical Branch, \\ Galveston, Texas \\ Address for correspondence Amna Qasim, MD, Department of \\ Pediatrics, University of Texas Medical Branch, 301 University \\ Boulevard, Galveston, TX-77555 (e-mail: amnahqasim@gmail.com).
}

Am J Perinatol Rep 2019;9:e121-e126.

\begin{abstract}
Introduction Congenital absence of the aortic valve leaflets is a rare association with hypoplastic left heart syndrome (HLHS).

Case A 37-year-old pregnant woman was referred for fetal evaluation of possible HLHS at 22 weeks of gestation. The fetal echocardiogram (ECHO) was remarkable for a hypoplastic left atrium, nearly atretic mitral valve, small left ventricle, and a hypoplastic aortic valve with severe aortic insufficiency. A female infant was born at term and postnatal ECHO confirmed the above findings. In addition, there was complete absence of the aortic valve leaflets. The patient underwent Norwood's procedure at day 5 of life with atrial septectomy, over-sewing of the aortic valve annulus, and a $4 \mathrm{~mm}$ Sano's shunt between the right ventricle and the

Keywords

- fetal echocardiography (ECHO)

- absent aortic valve

- hypoplastic left heart syndrome main pulmonary artery. She tolerated this surgery well and subsequently underwent a bidirectional Glenn's procedure at 8 months of life.

Conclusion Prenatal diagnosis of absent aortic valve should be suspected in the presence of severe aortic insufficiency in the fetal ECHO. Early postnatal intervention is critical as those patients are likely to deteriorate quickly. The over-sewing of the aortic valve may be important to prevent coronary steal and myocardial hypoperfusion which could potentially be detrimental.
\end{abstract}

Absence of the aortic valve (AAV) is a very rare congenital heart defect, unlike the absence of pulmonary valves. AAV is usually associated with other cardiac and non-cardiac anomalies. Most of the previously reported cases have been in spontaneously aborted fetuses, underscoring the high mortality of this heart defect.

\section{Case}

A 37-year-old pregnant female (Gravida 5, Para 3) was referred for fetal echocardiogram (ECHO) due to concerns

(-) Author's ORCID ID of Amna Qasim is orcid.org/0000-0002-0592-6705. (-) Author's ORCID ID of Ashraf M. Aly is orcid.org/0000-0001-9548-8034. of hypoplastic left heart (HLH) on anatomy ultrasound scan. Fetal ECHO at 22 weeks was significant for a hypoplastic left atrium, nearly atretic mitral valve, small nonapex forming left ventricle (LV), hypoplastic aortic valve with severe aortic insufficiency (-Fig. 1). Serial fetal ECHOs showed the same findings throughout pregnancy. No evidence of hydrops was seen. At 39-week gestation, a female infant was delivered with a birth weight $3.2 \mathrm{~kg}$ and Apgar's scores of 8 and 8 at 1 and 5 minutes, respectively. Prostaglandin E-1 $(0.05 \mathrm{mcg} / \mathrm{kg} /$ $\mathrm{min}$ ) infusion was started within an hour of delivery. Postnatal ECHO findings ( - Fig. 2, - Video 1) showed HLH variant with absent aortic valve and severe aortic insufficiency, a large PDA, moderate sized atrial septal defect (ASD), and poor left ventricular posterior wall function. The LV was diffusely echogenic but there was no clear endocardial fibro elastosis. received

September 12, 2018 accepted after revision December 2, 2018
DOI https://doi.org/

10.1055/s-0038-1677480. ISSN 2157-6998.
Copyright $\odot 2019$ by Thieme Medical Publishers, Inc., 333 Seventh Avenue, New York, NY 10001, USA. Tel: +1(212) 584-4662.
License terms

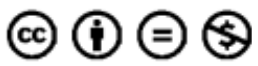




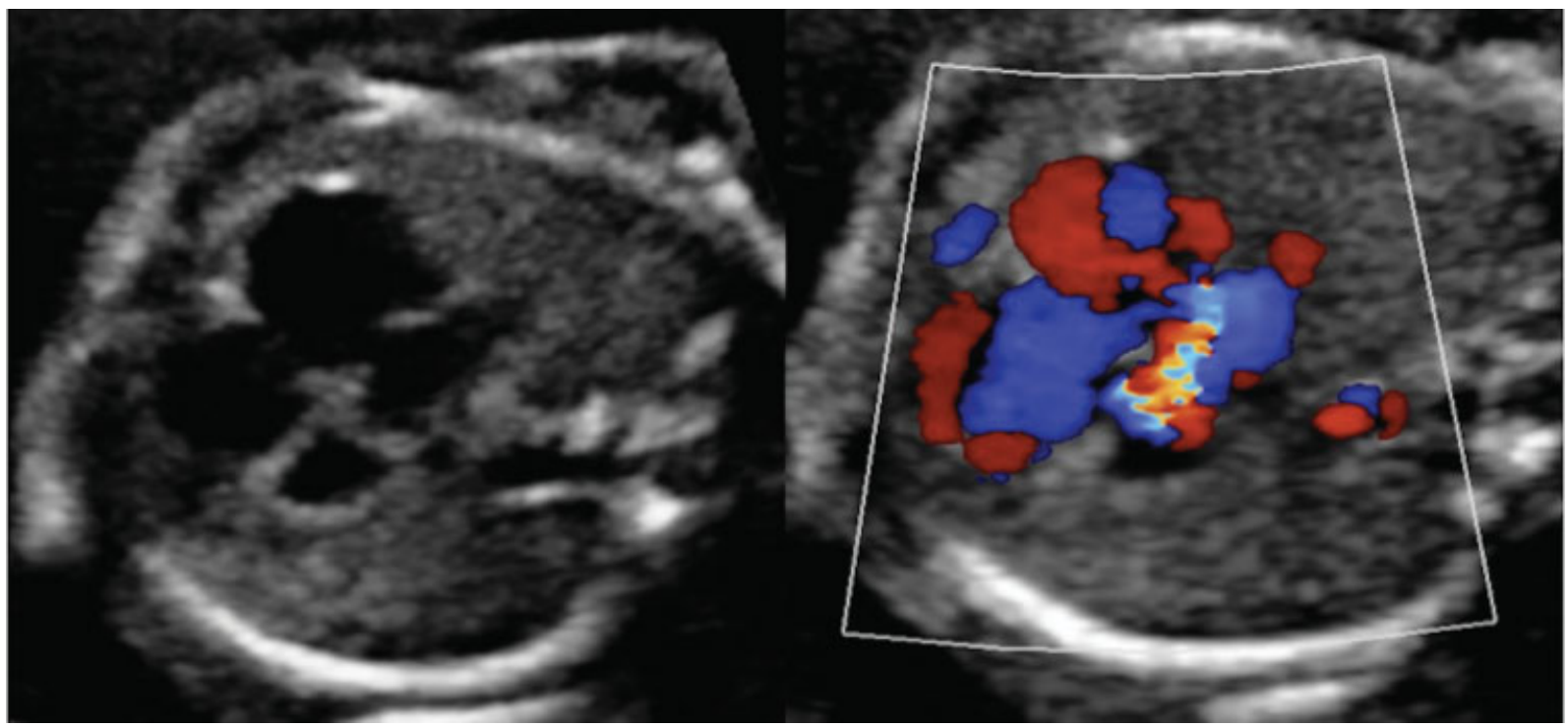

Fig. 1 Fetal ECHO "4-chamber view" showing a hypoplastic left ventricle and left atrium (left) and color Doppler's image showing aortic regurgitation (right).

There was decimal mitral inflow and a mild tricuspid valve insufficiency. The right ventricle was globular with a normal function. The coronary anatomy was normal but the flow was difficult to assess. Since the patient had no dysmorphic features or any extra cardiac anomaly, no genetic testing was done. The patient successfully underwent the first stage Norwood's procedure on the 5th day of life. The procedure included atrial septectomy, an end to side main pulmonary artery to aorta (Damus-Kaye-Stansel) anastamosis, PDA ligation, over-sewing of the aortic valve, and the placement of a $4 \mathrm{~mm}$ Sano's shunt between the right ventricle and the main pulmonary artery. Subsequently, she underwent the bidirectional Glenn's procedure at 8 months of life and is currently doing well and maintaining her $\mathrm{O}_{2}$ saturations in the mid-80s on room air.

\section{Video 1}

A parasternal long axis view showing a hypoplastic left ventricle and an absent aortic valve with severe regurgitation. Online content including video sequences viewable at: www.thieme-connect.com/ products/ejournals/html/10.1055/s-0038-1677480.

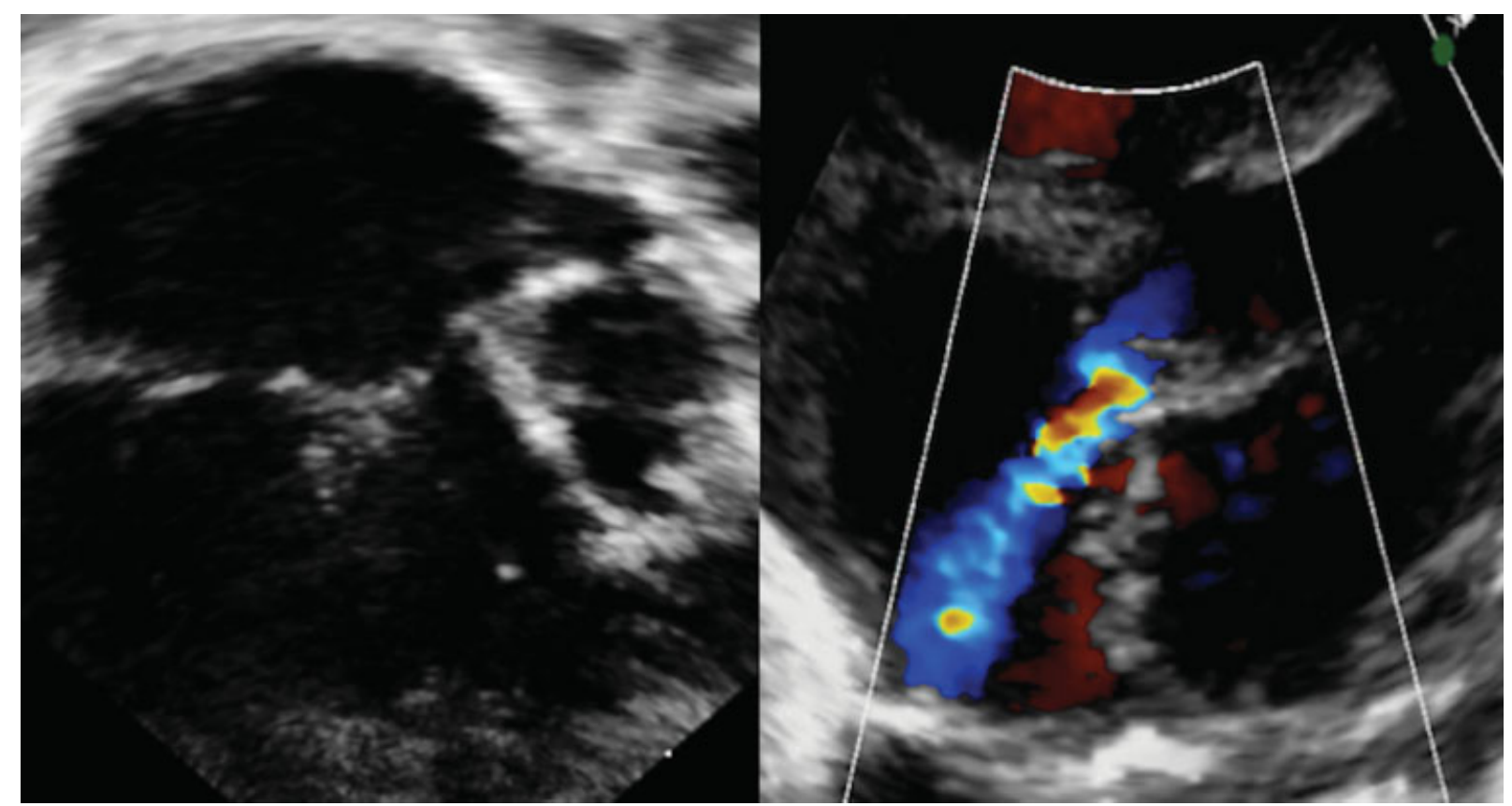

Fig. 2 Postnatal ECHO showing a hypoplastic left ventricle seen in 4-chamber view (left) and aortic regurgitation seen in parasternal long axis view (right). 


\section{Discussion}

Absent aortic valve (AAV) is a rare congenital heart defect with our case being the 26 th that was reported in literature. ${ }^{1}$ It is usually associated with other congenital anomalies including HLH, double outlet right ventricle (DORV), mitral atresia and absent, or dysplastic pulmonary valve. The clinical presentation may vary but mainly includes cyanosis, respiratory distress and cardiomegaly in the majority of reported cases. - Table 1 shows a review of previously

Table 1 Summary of all reported cases with absent aortic valves

\begin{tabular}{|c|c|c|c|c|c|c|}
\hline $\begin{array}{l}\text { Case no., } \\
\text { gender }\end{array}$ & $\begin{array}{l}\text { Diagnosis } \\
\text { age and } \\
\text { method }\end{array}$ & Other CHD & $\begin{array}{l}\text { Noncardiac } \\
\text { anomalies }\end{array}$ & $\begin{array}{l}\text { Clinical pre- } \\
\text { sentation }\end{array}$ & Outcome & Ref. \\
\hline $1, M$ & $\begin{array}{l}36 \mathrm{~h}, \\
\text { autopsy }\end{array}$ & $\begin{array}{l}\text { DORV, enlarged RA } \\
\text { and RV, ASD, PDA, } \\
\text { dysplastic LV, } \\
\text { hypoplastic MV and LV }\end{array}$ & Accessory spleen & $\begin{array}{l}\text { Severe cya- } \\
\text { nosis and } \\
\text { cardiome- } \\
\text { galy at } 36 \mathrm{~h}\end{array}$ & Death at $2 \mathrm{~d}$ & 7 \\
\hline $2, M$ & $\begin{array}{l}32 \text { wk, fetal } \\
\text { ECHO }+ \\
\text { autopsy }\end{array}$ & $\begin{array}{l}\text { DORV, common AV } \\
\text { canal, hypoplastic MV } \\
\text { and LV }\end{array}$ & $\begin{array}{l}\text { Absent R SVC, } \\
\text { anomalous L SVC, } \\
\text { splenic nodules, } \\
\text { gut malrotation }\end{array}$ & $\begin{array}{l}\text { Hydrops, } \\
\text { severe poly- } \\
\text { hydramnios, } \\
\text { RD }\end{array}$ & $\begin{array}{l}\text { Death at } \\
20 \mathrm{~h}\end{array}$ & 8 \\
\hline $3, M$ & $\begin{array}{l}24 \mathrm{~h}, \mathrm{ECHO} \\
+ \text { autopsy }\end{array}$ & $\begin{array}{l}\text { Hypoplastic MV and } \\
\text { LV, EFE of LV }\end{array}$ & None & $\begin{array}{l}\text { Cyanosis, } \\
\text { RD, } \\
\text { tachycardia }\end{array}$ & Death at $8 \mathrm{~d}$ & 9 \\
\hline $4, M$ & $\begin{array}{l}4 \mathrm{~h}, \mathrm{ECHO}+ \\
\text { autopsy }\end{array}$ & $\begin{array}{l}\text { Hypoplastic LA, EFE of } \\
\text { LV, anomalous RSA, } \\
\text { dysplastic TV and PV, } \\
\text { PDA }\end{array}$ & $\begin{array}{l}3 \text { lobes in left lung } \\
\text { and horseshoe } \\
\text { kidneys }\end{array}$ & $\begin{array}{l}\text { Severe cya- } \\
\text { nosis and RD }\end{array}$ & Death at $4 \mathrm{~d}$ & 10 \\
\hline $5, F$ & $1 \mathrm{~d}$, autopsy & $\begin{array}{l}\text { ASD, VSD, Interrupted } \\
\text { aortic arch, anomalous } \\
\text { RSA }\end{array}$ & $\begin{array}{l}\text { DiGeorge's syn- } \\
\text { drome (absent thy- } \\
\text { mus, PTH glands) }\end{array}$ & $\begin{array}{l}\text { RD, cardio- } \\
\text { megaly }\end{array}$ & $\begin{array}{l}\text { Death at } \\
36 \mathrm{~h}\end{array}$ & 11 \\
\hline $6, M$ & $\begin{array}{l}12 \mathrm{~h}, \mathrm{ECHO} \\
+ \text { autopsy }\end{array}$ & $\begin{array}{l}\text { MV atresia, Dysplastic } \\
\text { PV, PDA, normal LV }\end{array}$ & ND & RD, cyanosis & $\begin{array}{l}\text { Death at } \\
24 \mathrm{~h}\end{array}$ & 12 \\
\hline $7, M$ & $\begin{array}{l}11 \mathrm{~h}, \mathrm{ECHO} \\
+ \text { autopsy }\end{array}$ & Dysplastic MV, PDA & ND & $\begin{array}{l}\text { Cyanosis, } \\
\text { RDS }\end{array}$ & $\begin{array}{l}\text { Death at } \\
16 \mathrm{~h}\end{array}$ & 13 \\
\hline $8, M$ & $\begin{array}{l}20 \mathrm{~h}, \\
\text { autopsy }\end{array}$ & $\begin{array}{l}\text { Dysplastic MV, PV ste- } \\
\text { nosis, LV EFE }\end{array}$ & $\begin{array}{l}\text { Cortical renal cysts, } \\
\text { hydroureter/ } \\
\text { nephrosis, } \\
\text { microcephaly }\end{array}$ & $\begin{array}{l}\text { Cyanosis, } \\
\text { RD, cardio- } \\
\text { megaly }\end{array}$ & $\begin{array}{l}\text { Death at } \\
20 \mathrm{~h}\end{array}$ & 14 \\
\hline $9, M$ & $\begin{array}{l}24 \mathrm{~h}, \\
\text { autopsy }\end{array}$ & $\begin{array}{l}\text { Hypoplastic LA and LV, } \\
\text { LV EFE, ASD, PDA }\end{array}$ & $\begin{array}{l}\text { Hemosiderosis of } \\
\text { liver, minimal } \\
\text { deposits in kidney } \\
\text { and spleen }\end{array}$ & RD, cyanosis & $\begin{array}{l}\text { Death at } \\
24 \mathrm{~h}\end{array}$ & 14 \\
\hline $10, M$ & $4 \mathrm{~d}$, echo & $\begin{array}{l}\text { MV atresia, Ebstein } \\
\text { malformation, TAPVR, } \\
\text { PDA }\end{array}$ & $\begin{array}{l}\text { Hemosiderosis of } \\
\text { liver }\end{array}$ & Cyanosis & Death at $6 \mathrm{~d}$ & 14 \\
\hline $11, \mathrm{~F}$ & $\begin{array}{l}18 \mathrm{wk}, \\
\text { autopsy }\end{array}$ & $\begin{array}{l}\text { DORV, HLV, VSD, } \\
\text { straddling of TV, MV } \\
\text { atresia, absent PV }\end{array}$ & $\begin{array}{l}\text { Hypoplastic nose, } \\
\text { radial aplasia, } \\
\text { absent thumbs, } \\
\text { absent left index } \\
\text { finger, intestinal } \\
\text { malrotation, horse- } \\
\text { shoe kidney }\end{array}$ & $\begin{array}{l}\text { Spontaneous } \\
\text { abortion, } \\
\text { severely } \\
\text { macerated } \\
\text { fetus }\end{array}$ & IUD at $18 \mathrm{wk}$ & 15 \\
\hline $12, M$ & $\begin{array}{l}18 \mathrm{wk}, \\
\text { autopsy }\end{array}$ & $\begin{array}{l}\text { Absent PV, VSD, } \\
\text { small MV, thickened LV }\end{array}$ & $\begin{array}{l}\text { Cleft lip/palate, low } \\
\text { set ears }\end{array}$ & $\begin{array}{l}\text { Spontaneous } \\
\text { abortion, } \\
\text { severely } \\
\text { macerated } \\
\text { fetus }\end{array}$ & IUD at $18 \mathrm{wk}$ & 15 \\
\hline $13, \mathrm{~F}$ & $\begin{array}{l}18 \mathrm{wk}, \\
\text { autopsy }\end{array}$ & $\begin{array}{l}\text { Complete AVSD, per- } \\
\text { sistent LSVC, anoma- } \\
\text { lous RSA }\end{array}$ & $\begin{array}{l}\text { Nuchal bleb, } \\
\text { edema, thymic } \\
\text { hypoplasia, }\end{array}$ & $\begin{array}{l}\text { Generalized } \\
\text { edema }\end{array}$ & $\begin{array}{l}\text { Abortion at } \\
18 \mathrm{wk}\end{array}$ & 16 \\
\hline
\end{tabular}


Table 1 (Continued)

\begin{tabular}{|c|c|c|c|c|c|c|}
\hline $\begin{array}{l}\text { Case no., } \\
\text { gender }\end{array}$ & $\begin{array}{l}\text { Diagnosis } \\
\text { age and } \\
\text { method }\end{array}$ & Other CHD & $\begin{array}{l}\text { Noncardiac } \\
\text { anomalies }\end{array}$ & $\begin{array}{l}\text { Clinical pre- } \\
\text { sentation }\end{array}$ & Outcome & Ref. \\
\hline & & & $\begin{array}{l}\text { Trisomy } 13 \text { on } \\
\text { cytogenetics }\end{array}$ & & & \\
\hline $14, \mathrm{~F}$ & $\begin{array}{l}14 \mathrm{wk}, \\
\text { autopsy }\end{array}$ & $\begin{array}{l}\text { DORV, VSD, absent } \\
\text { PV, MV atresia, hypo- } \\
\text { plastic LA }\end{array}$ & $\begin{array}{l}\text { Cystic hygroma, } \\
\text { umbilical hernia- } \\
\text { tion, single UA, } \\
\text { thymic hypoplasia }\end{array}$ & - & $\begin{array}{l}\text { Artificial } \\
\text { abortion at } \\
14 \mathrm{wk}\end{array}$ & 16 \\
\hline $15, M$ & $\begin{array}{l}21 \mathrm{wk}, \\
\text { autopsy }\end{array}$ & DORV, VSD, absent PV & $\begin{array}{l}\text { Cystic hygroma, } \\
\text { single UA, absent } \\
\text { thymus }\end{array}$ & $\begin{array}{l}\text { Generalized } \\
\text { edema }\end{array}$ & $\begin{array}{l}\text { Artificial } \\
\text { abortion at } \\
21 \mathrm{wk}\end{array}$ & 16 \\
\hline 16, ND & $\begin{array}{l}17 \text { wk, fetal } \\
\mathrm{ECHO}+ \\
\text { autopsy }\end{array}$ & DORV, HLV, VSD, PDA & $\begin{array}{l}\text { Hydranencephaly, } \\
\text { hypoplastic left } \\
\text { forearm, right } \\
\text { radial aplasia }\end{array}$ & $\begin{array}{l}\text { Hydrops, } \\
\text { pericardial } \\
\text { effusion, car- } \\
\text { diomegaly }\end{array}$ & $\begin{array}{l}\text { Termination } \\
\text { at } 18 \mathrm{wk}\end{array}$ & 17 \\
\hline $17, M$ & $\begin{array}{l}9 \mathrm{~h}, 2 \mathrm{D} \\
\mathrm{ECHO}\end{array}$ & $\begin{array}{l}\text { VSD, patent LSVC, } \\
\text { absent MV, PDA, EFE of } \\
\text { LV }\end{array}$ & $\begin{array}{l}\text { High-arched palate, } \\
\text { low set ears }\end{array}$ & $\begin{array}{l}\text { Heart failure, } \\
\text { cyanosis }\end{array}$ & $\begin{array}{l}\text { Death at } \\
20 \mathrm{~h}\end{array}$ & 18 \\
\hline $18, M$ & $\begin{array}{l}12 \mathrm{~h}, \mathrm{ECHO} \\
+ \text { cath }\end{array}$ & $\begin{array}{l}\text { HLH, EFE of LV, dys- } \\
\text { plastic MV }\end{array}$ & ND & $\begin{array}{l}\text { Cyanosis, } \\
\text { cardiome- } \\
\text { galy }\end{array}$ & $\begin{array}{l}\text { Norwood's } \\
+ \text { BT shunt } \\
\text { at } 7 \text { dol, } \\
\text { death at } 20 \\
\text { dol }\end{array}$ & 19 \\
\hline 19, M & $\mathrm{ND}, \mathrm{ECHO}$ & $\begin{array}{l}\text { Dysplastic LV, MV } \\
\text { atresia, PDA }\end{array}$ & None & $\begin{array}{l}\text { Mild } \\
\text { cyanosis }\end{array}$ & $\begin{array}{l}\text { Norwood's } \\
\text { at } 12 \text { dol, } \\
\text { heart trans- } \\
\text { plant at } 1.5 \\
\text { mo }\end{array}$ & 3 \\
\hline $20, M$ & $\begin{array}{l}\text { Fetal ECHO } \\
\text { at } 29 \text { wk }\end{array}$ & $\begin{array}{l}\text { VSD, PDA, dysplastic } \\
\text { PV }\end{array}$ & ND & $\begin{array}{l}\text { Fetal } \\
\text { hydrops }\end{array}$ & Death at $4 \mathrm{~h}$ & 20 \\
\hline $21, M$ & $2 \mathrm{~d}, \mathrm{ECHO}$ & MV atresia & ND & $\begin{array}{l}\text { Cyanosis, } \\
\text { Respiratory } \\
\text { distress }\end{array}$ & $\begin{array}{l}\text { Norwood's } \\
\text { at } 9 \mathrm{~d}, \mathrm{BDG} \\
\text { at } 6 \text { mo, } \\
\text { Fontan's at } \\
6 \mathrm{y}\end{array}$ & 2,21 \\
\hline $22, \mathrm{ND}$ & $\begin{array}{l}31 \text { wk, Fetal } \\
\text { ECHO }\end{array}$ & $\begin{array}{l}\text { LV hypertrophy and } \\
\text { EFE }\end{array}$ & $\begin{array}{l}\text { Generalized lym- } \\
\text { phangiectasis, sys- } \\
\text { temic air embolism }\end{array}$ & $\begin{array}{l}\text { Fetal } \\
\text { hydrops }\end{array}$ & Death at $2 \mathrm{~h}$ & 22 \\
\hline $23, \mathrm{~F}$ & $\begin{array}{l}24 \text { wk, Fetal } \\
\text { ECHO }\end{array}$ & $\begin{array}{l}\text { DORV, large VSD, left } \\
\text { SVC, AP window }\end{array}$ & $\begin{array}{l}\text { Cystic hygroma, } \\
\text { cerebral ventriculo- } \\
\text { megaly, microce- } \\
\text { phaly, clinodactyly, } \\
\text { bilateral ear } \\
\text { dysplasia, choanal } \\
\text { atresia }\end{array}$ & $\begin{array}{l}\text { Respiratory } \\
\text { depression }\end{array}$ & Death at $2 \mathrm{~d}$ & 23 \\
\hline $24, M$ & $\begin{array}{l}24 \text { wk, Fetal } \\
\text { ECHO }\end{array}$ & HLH, MV atresia & Broad fingers & $\begin{array}{l}\text { Cardiome- } \\
\text { galy, } \\
\text { cyanosis }\end{array}$ & $\begin{array}{l}\text { Norwood's } \\
\text { and BDG at } 3 \\
\text { mo, death at } \\
10 \text { mo }\end{array}$ & 1 \\
\hline $25, M$ & $\begin{array}{l}23 \text { wk, Fetal } \\
\text { ECHO }\end{array}$ & $\begin{array}{l}\text { Dilated LV, EFE, hypo- } \\
\text { plastic MV }\end{array}$ & ND & $\begin{array}{l}\text { Fetal } \\
\text { hydrops }\end{array}$ & IUD at $28 \mathrm{w}$ & 1 \\
\hline
\end{tabular}

Abbreviations: AAV, absent aortic valve; AP, aorticopulmonary; ASD, atrial septal defect; AV, atrioventricular; AVSD, atrioventricular septal defect; BDG, bi-directional Glenn; CHD, congenital heart disease; d, day; dol, day of life; DORV, double outlet right ventricle; ECHO, echocardiogram; EFE, endocardial fibroelastosis; F, female; h, hour; HLH, hypoplastic left heart; HLV, hypoplastic left ventricle; IUD, intrauterine demise; L, left; LV, left ventricle; $M$, male, mo, months; MV, mitral valve; ND, not described; PDA, patent ductus arteriosus; PTH, parathyroid glands; PV, pulmonary valve; R, right; RA, right atrium; RD, respiratory distress; RSA, right subclavian artery; RV, right ventricle; SVC, superior vena cava; TAPVR, total anomalous pulmonary venous return; TV, tricuspid valve; UA, umbilical artery; wk, week; y, year. 
reported cases to date. The rarity of this condition may be due to the high rate of mortality leading to spontaneous abortions. Even though it was initially thought to be an Xlinked recessive condition due to the first few reports being males, that does not seem to be the case since a few female cases have been reported.

Only two reported cases have survived beyond the first few days of life. Harada et $\mathrm{al}^{2}$ described the first case of successful palliative surgery in a patient with HLH syndrome and AAV. At 9 days of life, the patient underwent Norwood's procedure with a Blalock-Taussig (BT) shunt. The aortic valve was noted to be absent and left ventricular cavity size was small with endocardial fibroelastosis. The patient did well and underwent the bidirectional Glenn's procedure at the age of 6 months and the Fontan's procedure at the age of 6 years. The aortic annulus was not oversewn with the thought that coronary circulation would be maintained owing to the markedly diminished size and compliance of the LV. In addition, a balloon occlusion of the left ventricular outflow tract showed no effect on the coronary circulation.

The second case of AAV that survived beyond the first few days of life was reported by Krasemann et al. ${ }^{3}$ Associated cardiac defects included mitral atresia and a large noncontractile hypoplastic LV. On day 12 of life the infant underwent the Norwood's procedure with additional surgical closure of the aortic valve with the goal of preservation of coronary perfusion. The postoperative course was complicated by difficulty weaning from mechanical ventilation. An orthotropic heart transplantation was successfully performed 4 weeks after the Norwood's procedure. The postoperative course was uneventful. The child was doing well at 5 months of age when this case was reported.

The majority of reported cases of AAV were diagnosed either on autopsy or on postnatal ECHO, with only a handful of cases diagnosed on fetal ECHO ( - Table 1). Fetal ECHO was introduced as a diagnostic modality in the early 1970s and has advanced significantly over the past few decades. ${ }^{4}$ It has been shown to be a valuable tool in the optimization of perinatal care. ${ }^{5,6}$ To date, no fetal interventions have been attempted for the palliation of this condition.

Our case is one of the first few reported cases that were diagnosed by fetal ECHO, the second case that underwent successful palliative repair, and the third case that survived beyond the first few days of life. To our knowledge, it is also the first reported case in which the infant underwent successful Norwood's procedure with closure of the aortic valve annulus. Closure of the aortic valve annulus would prevent the development of coronary steal syndrome that may occur if continued aortic regurgitation leads to increased left ventricular size and compliance.

\section{Conclusion}

AAV is rare and is usually associated with other congenital cardiac anomalies, especially HLHS. It should be suspected in the presence of aortic insufficiency on fetal ECHO. Palliative repair with Norwood's procedure with over-sewing of the aortic annulus could potentially prevent coronary steal and myocardial hypoperfusion in these patients.

\section{Ethical Approval}

This article does not contain any studies with human participants performed by any of the authors.

\section{Informed Consent}

Informed consent was waived for this study since no identifying information was included.

\section{Conflict of Interest}

The authors declare that they have no conflicts of interest.

\section{References}

1 Murakami T, Horigome H, Shiono J, et al. Prenatal diagnosis of congenital absence of aortic valve: a report of two cases with different outcomes and a literature review. Fetal Diagn Ther 2015; 38(04):307-314

2 Harada Y, Takeuchi T, Satomi G, Yasukouchi S. Absent aortic valve: successful palliation in the neonate. Ann Thorac Surg 1998;66 (03):935-936

3 Krasemann T, Kehl HG, Hammel D, Asfour B. Congenital aortic regurgitation due to absent aortic cusps and high-degree mitral stenosis. Pediatr Cardiol 2003;24(03):304-306

4 Maulik D, Nanda NC, Maulik D, Vilchez G. A brief history of fetal echocardiography and its impact on the management of congenital heart disease. Echocardiography 2017;34(12): $1760-1767$

5 Liu H, Zhou J, Feng QL, et al. Fetal echocardiography for congenital heart disease diagnosis: a meta-analysis, power analysis and missing data analysis. Eur J Prev Cardiol 2015;22(12): 1531-1547

6 Holland BJ, Myers JA, Woods CR Jr. Prenatal diagnosis of critical congenital heart disease reduces risk of death from cardiovascular compromise prior to planned neonatal cardiac surgery: a meta-analysis. Ultrasound Obstet Gynecol 2015;45(06): 631-638

7 Toews WH, Lortscher RH, Kelminson LL. Double outlet right ventricle with absent aortic valve. Chest 1975;68(03):381-382

8 Bierman FZ, Yeh MN, Swersky S, Martin E, Wigger JH, Fox H. Absence of the aortic valve: antenatal and postnatal two-dimensional and Doppler echocardiographic features. J Am Coll Cardiol 1984;3(03):833-837

9 Rossi MB, Ho SY, Tasker RC. Absent aortic valve leaflets. Int J Cardiol 1986;11(02):235-237

10 Niwa K, Ikeda F, Miyamoto H, Nakajima H, Ando M. Absent aortic valve with normally related great arteries. Heart Vessels 1987;3 (02):104-107

11 Weintraub RG, Chow CW, Gow RM. Absence of the leaflets of the aortic valve in DiGeorge syndrome. Int J Cardiol 1989;23(02): 255-257

12 Cabrera A, Galdeano JM, Pastor E. Absence of the aortic valve cusps with mitral atresia, normal left ventricle, and intact ventricular septum. Br Heart J 1990;63(03):187-188

13 Parikh SR, Hurwitz RA, Caldwell RL, Waller B. Absent aortic valve in hypoplastic left heart syndrome. Am Heart J 1990;119(04): 977-978

14 Lin AE, Chin AJ. Absent aortic valve: a complex anomaly. Pediatr Cardiol 1990;11(04):195-198

15 Hartwig NG, Vermeij-Keers C, De Vries HE, Gittenberger-De Groot AC. Aplasia of semilunar valve leaflets: two case reports and developmental aspects. Pediatr Cardiol 1991;12(02): 114-117 
16 Miyabara S, Ando M, Yoshida K, Saito N, Sugihara H. Absent aortic and pulmonary valves: investigation of three fetal cases with cystic hygroma and review of the literature. Heart Vessels 1994;9 (01):49-55

17 Marek J, Skovránek J, Povýsilová V. Congenital absence of aortic and pulmonary valve in a fetus with severe heart failure. Heart 1996;75 (01):98-100

18 Nakagawa M, Okamoto N, Fujino H, et al. Doppler echocardiographic evaluation of the hemodynamics in absent aortic valve. Can J Cardiol 1999;15(11):1283-1286

19 Rouillard KP, Moore P, Silverman NH. Congenital absence of aortic valvar leaflets: a rare variant of the hypoplastic left heart syndrome. Cardiol Young 2001;11(04):453-457
20 Eronen M, Heikkilä P. Absent aortic and dysplastic pulmonary valves associated with ventricular septal defect in fetal hydrops. Pediatr Cardiol 2003;24(04):400-402

21 Hibino N, Harada Y, Hiramatsu T, Yasukochi S, Satomi G. Fontan operation for hypoplastic left heart syndrome with absent aortic valve. J Thorac Cardiovasc Surg 2004;128(02):315-316

22 Muneuchi J, Kuraoka A, Ochiai Y, Nishibatake M, Sese A, Joo K. Fatal systemic air embolism in a neonate with absent aortic valve. Pediatr Cardiol 2011;32(06):839-841

23 Sabati AA, Wong PC, Randolph L, Pruetz JD. Absent aortic valve associated with double outlet right ventricle and aortopulmonary window: physiologic implications of a rare malformation in both the fetus and neonate. Congenit Heart Dis 2014;9(03):E98-E104 